\title{
RELATO DE CASO \\ RELATO DE CASO: LUXAÇÃO TESTICULAR POR TRAUMA
} CASE REPORT: TRAUMATIC TESTICULAR DISLOCATION

Gustavo Simon Mendes Ruiz ${ }^{1}$, Giovanni Vitor Garabini ${ }^{1}$, Paulo César Vieira dos Santos ${ }^{1}$, Giovanni Montinni Sandoval ${ }^{2}$

\section{ด ACESSO LIVRE}

Citação: Ruiz GSM, Garabini GV, Santos PCV, Sandoval GM (2021) RELATO DE CASO: LUXAÇÃO TESTICULAR POR TRAUMA. Revista de Patologia do Tocantins, 7(4):

\section{Instituição:}

${ }^{1}$ Acadêmicos de medicina da universidade Federal do Tocantins (UFT) - Palmas, Tocantins, Brasil.

${ }^{2}$ Médico especialista em Urologia pela Santa Casa de Misericórdia de Goiânia, Goiás, Brasil - 1991

\section{Autor correspondente:}

Gustavo Simon Mendes Ruiz, gustavosimon2006@gmail.com

Editor: Carvalho A. A. B. Medicina, Universidade Federal do Tocantins, Brasil.

Publicado: 23 de fevereiro de 2021.

Direitos Autorais: (c) 2021 Ruiz et al. Este é um artigo de acesso aberto que permite o uso, a distribuição e a reprodução sem restrições em qualquer meio, desde que o autor original e a fonte sejam creditados.

Conflito de interesses: os autores declararam que não existem conflitos de interesses.

\begin{abstract}
RESUMO
Introdução: A luxação testicular após trauma perineal contuso é um evento raro, que ocorre principalmente em acidentes de motocicleta e traumas por montaria em cavalos. Devido à sua raridade e por estar geralmente associada com outras lesões graves, pode haver demora em seu diagnóstico e tratamento. O diagnóstico pode ser estabelecido no exame físico com a ausência de testículo no saco escrotal. Tomografia computadorizada (TC) e ultrassom com doppler também são ferramentas úteis no diagnóstico dessa condição. $O$ tratamento pode ser realizado com redução manual ou cirurgia. Descrição do Caso: MJFS, masculino, vítima de acidente motociclístico com trauma contuso de pelve e membros inferiores. Durante a avaliação inicial foi constatada fratura de membro inferior esquerdo e disjunção da sínfise púbica. O paciente foi submetido a alinhamento do membro inferior e optou-se pelo tratamento conservador da disjunção púbica. Após um mês do acidente o paciente relata dor pélvica à esquerda. Ao exame físico foi constatada a ausência do testículo na bolsa escrotal esquerda com massa palpável dolorosa na região pélvica esquerda. Foi realizada então uma TC de pelve que mostrou o testículo esquerdo ectópico em tecido subcutâneo externo ao canal inguinal esquerdo. MJFS foi submetido à cirurgia para liberação e inserção do testículo na bolsa escrotal. O procedimento se deu sem intercorrências e o paciente recebeu alta em bom estado geral. Discussão: A luxação testicular é um evento facilmente detectado em exame físico. Pode provocar complicações como ruptura testicular, atrofia testicular, malignidade futura, infertilidade e disfunção endócrina, além de poder se associar com outras lesões como as de trato urinário. Conclusões: Dessa forma, é importante estar atento para sua ocorrência diante de um trauma pélvico.

Palavras-chave: Testículo; Ferimentos e Lesões; Procedimentos Cirúrgicos Urológicos; Fraturas do Quadril.
\end{abstract}

\begin{abstract}
Introduction: Testicular dislocation after blunt perineal trauma is a rare event, which occurs mainly in motorcycle accidents and horse riding trauma. Due to its rarity and because it is usually associated with other serious injuries, there may be a delay in its diagnosis and treatment. The diagnosis can be established on physical examination with the absence of a testicle in the scrotum. Computed tomography (CT) and doppler ultrasound are also useful tools in the diagnosis of this condition. Treatment can be performed with manual reduction or surgery. Case Description: MJFS, male, victim of a motorcycle accident with blunt trauma to the pelvis and lower limbs. During the initial evaluation, a fracture of the left lower limb and disjunction of the pubic symphysis were observed. The patient underwent lower limb alignment and a conservative treatment of pubic disjunction was chosen. One month after the accident, the patient reports left pelvic pain. Physical examination revealed the absence of a testicle in the left scrotum with a painful palpable mass in the left pelvic region. A pelvic CT scan was then performed which showed the ectopic left testicle in subcutaneous tissue external to the left inguinal canal. MJFS underwent surgery to release and insert the testicle into the scrotum. The procedure was uneventful and the patient was discharged in good general condition. Discussion: Testicular dislocation is an event easily detected on physical examination. It can cause complications such as testicular rupture, testicular atrophy, future malignancy, infertility and endocrine dysfunction, in addition to being associated with other lesions such as urinary tract injuries. Conclusions: Thus, it is important to be aware of its occurrence in the face of a pelvic trauma.
\end{abstract}

Keywords: Testis; Wounds and Injuries; Urologic Surgical Procedures; Hip Fractures. 


\section{INTRODUÇÃO}

A luxação testicular ou criptorquidismo adquirido é uma apresentação clínica rara, com poucos relatos na literatura e que ocorre mais comumente como resultado de um traumatismo escrotal. Estes relatos são mais frequentes em acidentes de motocicleta, por contusão direta contra o tanque de gasolina ou sobre o guidão, e traumas por montaria em cavalos. $^{1-3}$ Como a luxação testicular traumática pode estar associada a outras lesões graves, geralmente é um achado tardio, levando à demora do diagnóstico e tratamento. ${ }^{4}$

O diagnóstico de uma luxação testicular é frequentemente estabelecido durante o exame físico, com o achado de um hemiescroto vazio na palpação da bolsa escrotal. ${ }^{5}$ Ultrassonografia com doppler colorido e Tomografia computadorizada (TC) são as modalidades de imagem mais úteis para confirmar o diagnóstico. ${ }^{5-7} \mathrm{~A}$ ultrassonografia com doppler além de documentar a localização ectópica, serve para avaliar lesões associadas e eventual comprometimento vascular testicular. A tomografia computadorizada também pode ser usada em casos limítrofes de localização profunda inguinal. .,6,8,9 $^{\circ}$ diagnóstico e o tratamento precoces são recomendados para preservar a função testicular e evitar o risco de transformação maligna. ${ }^{4}$

Com relação ao tratamento, uma vez feito o diagnóstico é possível tentar uma redução rigorosa sob anestesia com a orientação de ultrassom Doppler para verificar a localização testicular e descartar a torção. ${ }^{10} \mathrm{Em}$ caso de dúvida, a abordagem cirúrgica é preferível. Mesmo em pacientes operados precocemente, um rápido desenvolvimento de adesão pode ocorrer. Recomenda-se a dissecção cuidadosa e a liberação dessas adesões com realocação e fixação do testículo no saco escrotal. ${ }^{2,4,11}$

\section{DESCRIÇÃO DO CASO}

MJFS, 27 anos, estudante. Vítima de acidente motociclístico - colisão moto carro com trauma de membros inferiores e pelve. Ao exame inicial no Pronto socorro foram constatadas: Disjunção de sínfise púbica e fratura da perna esquerda, e então o paciente ficou aos cuidados da ortopedia. Para a fratura da perna foi realizado o devido alinhamento, enquanto para a disjunção pélvica não havia necessidade de intervenção cirúrgica. 30 dias após o acidente o paciente relatou queixa de dor pélvica à esquerda com piora à palpação. Ao exame físico foi detectada massa pélvica dolorosa à esquerda e ausência do testículo esquerdo em região escrotal. $O$ paciente negou qualquer histórico de criptorquidia prévia. Foi requisitada então avaliação da equipe urológica que optou por realizar inicialmente, junto ao exame físico, uma ultrassonografia de bolsa escrotal que evidenciou uma ectopia testicular. A imagem do USG sugeria alojamento testicular em canal inguinal esquerdo e ao Doppler apresentava baixa perfusão sanguínea. Optou-se então por realizar uma tomografia (imagem 1) para melhor avaliação e tomada de conduta que confirmou os achados do USG.
Como conduta foi tentada inicialmente a reversão do testículo por manobras manuais com anestesia local, porém, sem sucesso terapêutico. Por conseguinte, foi instaurada terapia cirúrgica. O procedimento adotado foi uma inguinotomia à esquerda. Após a incisão foi encontrado o testículo aderido ao subcutâneo, exterior ao canal inguinal, com boa perfusão sanguínea, sem sinais de necrose evidentes. Optou-se então pela dilatação do canal testicular até a bolsa escrotal, liberação do testículo e reinserção do mesmo em bolsa escrotal com rafia. O procedimento se deu sem intercorrências. No pós-operatório paciente referiu melhora da dor, com testículo tópico e recebeu alta após 5 dias da cirurgia.

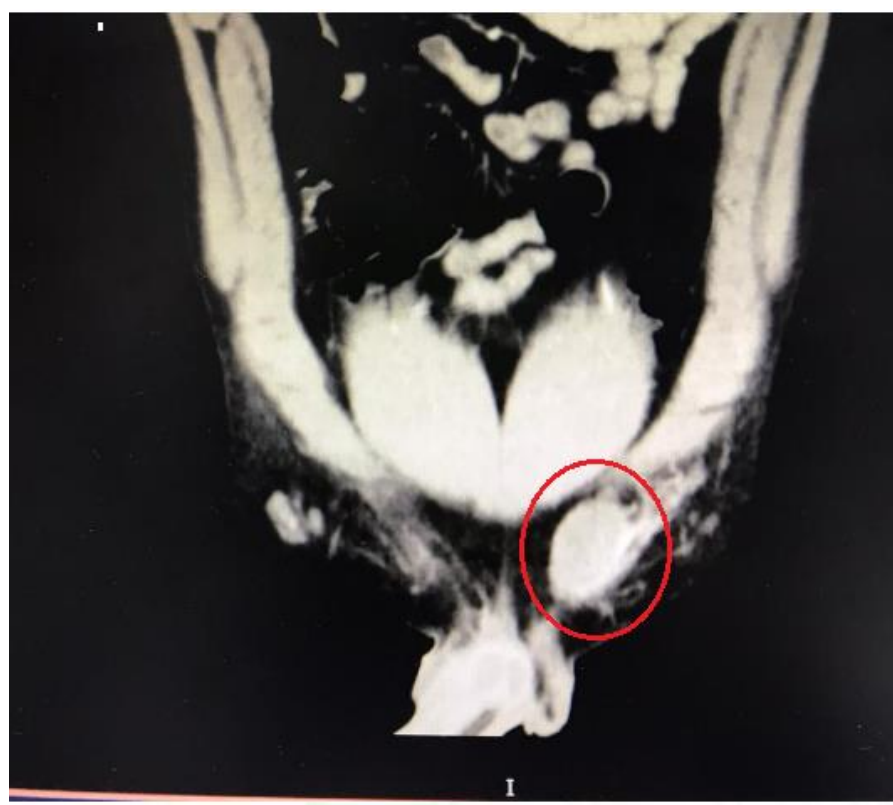

Imagem 1: Tomografia de pelve pré cirúrgica: testículo ectópico circulado em vermelho.

\section{DISCUSSÃO}

A luxação traumática do testículo é uma complicação rara de trauma perineal contuso. Mais comumente relatado em lesões causadas por acidentes de moto, no qual o tanque de combustível ou o guidão atinge o escroto e o períneo. 2,4,10,12 É frequentemente associado a múltiplas lesões, geralmente fraturas pélvicas e ruptura testicular, ${ }^{5}$ portanto, um diagnóstico ou tratamento tardio da luxação testicular não é incomum. ${ }^{4}$

A luxação testicular pode ser classificada em superficial ou interna. O testículo é mais comumente luxado para regiões superficiais como a fáscia abdominal, dentro da fáscia de Camper, regiões inguinais, abdominal inferior, pubiana ou peniana, sendo mais frequentemente luxado para a região inguinal superficial. ${ }^{5,7,10}$ As luxações internas são mais raras e ocorrem quando o testículo é forçado através do canal inguinal para a cavidade abdominal por força posterior ou devido a ruptura da bacia pélvica secundário a uma fratura pélvica. São geralmente associadas com anomalias como um anel inguinal externo dilatado, uma hérnia inguinal 
preexistente ou uma anormalidade testicular como testículos pequenos ou atrofiados. ${ }^{7,10} \mathrm{O}$ mecanismo mais comum das luxações testiculares parece ser a projeção do testículo através da fáscia espermática externa, cremastérica e interna. Geralmente, o ducto deferente, veias espermáticas e artéria testicular são deslocados juntamente com o testículo. Além disso, a túnica albugínea permanece intacta. Como a força da pressão é usada para deslocar o testículo, a túnica não é interrompida e não há ruptura testicular. ${ }^{5}$

O diagnóstico pode ser constatado durante o exame físico com achado de um hemiescroto vazio na palpação da bolsa escrotal. ${ }^{5}$ Porém, alguns fatores podem tornar o diagnóstico no exame clínico inicial mais difícil, como obesidade ou hematoma escrotal relacionado a lesão. ${ }^{7}$ É possível, no entanto, que muitos dos casos não diagnosticados sejam devido ao exame clínico inadequado ou dificultado pelas frequentes fraturas pélvicas associadas. Ultrassonografia ou TC são as modalidades de imagem mais úteis para confirmar a diagnóstico. A ultrassonografia com doppler além de documentar a localização ectópica, serve para avaliar lesões associadas e eventual comprometimento vascular testicular, que felizmente raramente ocorre. A tomografia computadorizada também pode ser usada em casos limítrofes de localização profunda inguinal. , $^{5,6,8,9}$

As lesões do trato urinário são frequentes nos casos de fratura do anel pélvico e estão frequentemente associadas a lesões na uretra ou na bexiga, além de lesões no pênis menos comumente..$^{5,9,13} \mathrm{O}$ paciente deste relato não apresentou queixas ou sinais de lesão de trato urinário.

Embora não seja uma ameaça aguda à vida, a luxação do testículo confere um risco aumentado de atrofia testicular, malignidade futura, infertilidade e disfunção endócrina. Essas alterações são geralmente reversíveis, desde que também não esteja presente a torção ou ruptura do testículo. ${ }^{5}$ Sakamoto et al. e Yoshimura et al. descreveram casos em que a espermatogênese foi restaurada em pacientes com luxação testicular após orquidopexia até 13 e 15 anos após a luxação. ${ }^{4,14}$ Isso sugere que, embora o diagnóstico de luxação seja crítico para evitar lesões iatrogênicas durante o tratamento cirúrgico das lesões pélvicas, o tratamento definitivo pode potencialmente ser adiado até que outras lesões sejam tratadas. ${ }^{5}$

Dessa forma, entende-se que luxação testicular é uma complicação rara de trauma escrotal contuso, geralmente ocorrendo após um acidente de motocicleta, pelo trauma direto da pelve com o tanque de combustível ou guidão. ${ }^{1,3-5,10}$ Profissionais que prestam atendimento de emergência devem estar cientes de sua ocorrência diante de qualquer trauma pélvico violento. ${ }^{2}$ Um exame meticuloso do escroto é recomendado, especialmente na presença de múltiplas lesões. USG e USG com Doppler colorido são as ferramentas mais úteis na avaliação de uma luxação testicular, enquanto uma tomografia computadorizada pode ser útil no caso de um trauma complexo. ${ }^{3,8,9}$
2018;2018:7162351.

https://doi.org/10.1155/2018/7162351

2. Gómez RG, Storme O, Catalán G, Marchetti $P$, Djordjevic M. Traumatic testicular dislocation. Int. Urol. Nephrol. 2014;46(10):1883-1887.

3. Feder M, Sacchetti A, Myrick S. Testicular dislocation following minor scrotal trauma. Am J Emerg Med. 1991;9(1):40-42. doi:10.1016/0735-6757(91)90012-9 4. Sakamoto $H$, Iwasaki S, Kushima M, Shichijo T. CASE REPORT Traumatic bilateral testicular dislocation : a recovery of spermatogenesis by orchiopexy 15 years after the onset. Fertil. Steril. 2009;90(5):2009-2011.

5. Middleton AH, Martin JM, Wittmann TA, Schmeling GJ. Testicular Dislocation after Pelvic Ring Injury: A Report of 2 Cases. JBJS Case Connect. 2019;9(4):2-7.

6. Ezra N, Afari A, Wong J. Pelvic and scrotal trauma: CT and triage of patients. Abdom Imaging. 2009;34(4):541544. doi:10.1007/s00261-008-9417-3

7. Ko SF, Ng SH, Wan YL, Huang CC, Lee TY, Kung C Te, et al. Testicular Dislocation: An Uncommon and Easily Overlooked Complication of Blunt Abdominal Trauma. Ann. Emerg. Med. 2004;43(3):371-375.

8. Weatherspoon K, Polansky S, Catanzano T. Ultrasound Emergencies of the Male Pelvis. Semin. Ultrasound, CT, MRI. 2017;38(4):327-344.

9. Kim W, Lee S, Jang H, Kim D. Delayed detection of testicular dislocation with pelvic ring fracture: A case report. J. Orthop. Sci. 2015;6-8.

10. Zavras N, Siatelis A, Misiakos E, Bagias G, Papachristos V, Machairas A. Testicular Dislocation After Scrotal Trauma: A Case Report and Brief Literature Review. Urol. Case Reports. 2014;2(3):101-104.

11. Phuwapraisirisan S, Lim M, Suwanthanma W. Surgical reduction in a delayed case of traumatic testicular dislocation. J. Med. Assoc. Thai. 2010 Nov;93(11):13171320.

12. Bhullar, Jasneet \& cheung, edmund. (2013). Acquired cryptorchidism in an ectopic location. International Journal of Case Reports and Images (IJCRI). 3. 21-23. 10.5348/ijcri-2012-08-159-CR-6.

13. Boudissa M, Ruatti S, Maisse N, Arvin-berod A, Trilling B. Bilateral testicular dislocation with pelvic ring fracture: A case report and literature review. Orthop. Traumatol. Surg. Res. 2013;99(4):485-487.

14. Yoshimura K, Okubo K, Ichioka K, Terada N, Matsuta $Y$, Arai $Y$. Restoration of spermatogenesis by orchiopexy 13 years after bilateral traumatic testicular dislocation. J. Urol. 2002 Feb;167(2 Pt 1):649-650.

\section{REFERÊNCIAS}

1. Carvalho NMN de, Marques ACX, Souza IT de, Soares VG, Nascimento FG do, Pinto LM, et al. Bilateral Traumatic Testicular Dislocation. Case Rep. Urol. [Internet]. 